\title{
Micromolar sodium fluoride mediates anti-osteoclastogenesis in Porphyromonas gingivalis-induced alveolar bone loss
}

\author{
Ujjal K Bhawal $^{1,2,3}$, Hye-Jin Lee ${ }^{4}$, Kazumune Arikawa ${ }^{2,5}$, Michiharu Shimosaka ${ }^{2,6}$, Masatoshi Suzuki ${ }^{2,6}$, \\ Toshizo Toyama ${ }^{7}$, Takenori Sato ${ }^{7}$, Ryota Kawamata $^{8}$, Chieko Taguchi $^{2,5}$, Nobushiro Hamada ${ }^{7}$, Ikuo Nasu ${ }^{2,5}$, \\ Hirohisa Arakawa ${ }^{3}$ and Koh Shibutani ${ }^{2,5}$
}

Osteoclasts are bone-specific multinucleated cells generated by the differentiation of monocyte/macrophage lineage precursors. Regulation of osteoclast differentiation is considered an effective therapeutic approach to the treatment of bone-lytic diseases. Periodontitis is an inflammatory disease characterized by extensive bone resorption. In this study, we investigated the effects of sodium fluoride (NaF) on osteoclastogenesis induced by Porphyromonas gingivalis, an important colonizer of the oral cavity that has been implicated in periodontitis. NaF strongly inhibited the $P$. gingivalis-induced alveolar bone loss. That effect was accompanied by decreased levels of cathepsin K, interleukin (IL)-1ß, matrix metalloproteinase 9 (MMP9), and tartrate-resistant acid phosphatase, which were up-regulated during $P$. gingivalis-induced osteoclastogenesis. Consistent with the in vivo anti-osteoclastogenic effect, $\mathrm{NaF}$ inhibited osteoclast formation caused by the differentiation factor RANKL (receptor activator of nuclear factor $\mathrm{\kappa B}$ ligand) and macrophage colony-stimulating factor (M-CSF). The RANKL-stimulated induction of the transcription factor nuclear factor of activated T cells (NFAT) $\mathrm{c} 1$ was also abrogated by NaF. Taken together, our data demonstrate that NaF inhibits RANKL-induced osteoclastogenesis by reducing the induction of NFATc1, ultimately leading to the suppressed expression of cathepsin K and MMP9. The in vivo effect of $\mathrm{NaF}$ on the inhibition of $\boldsymbol{P}$. gingivalis-induced osteoclastogenesis strengthens the potential usefulness of $\mathrm{NaF}$ for treating periodontal diseases.

International Journal of Oral Science (2015) 7, 242-249; doi:10.1038/ijos.2015.28; published 18 September 2015

Keywords: alveolar bone loss; osteoclasts; Porphyromonas gingivalis; sodium fluoride

\section{INTRODUCTION}

The regulation of bone metabolism results from a balance between bone resorption caused by osteoclasts and bone formation caused by osteoblasts. Sodium fluoride $(\mathrm{NaF})$ has been shown to influence the differentiation process of bone-forming cells. Several studies showed an increased alkaline phosphatase activity in osteoblastic cells in response to fluoride. ${ }^{1}$ Fluoride behaves as a cumulative element that can alter the accretion and resorption of bone tissues as well as affect the homeostasis of bone mineral metabolism. ${ }^{2-3}$ At lower doses, fluoride can lead to an increase in bone mass and has been investigated as a therapeutic agent for the treatment of postmenopausal osteoporosis in adults. ${ }^{4}$ Low fluoride doses promote osteoblast proliferation, stimulating bone formation in vitro and in vivo. ${ }^{5-6}$

Periodontal disease has an adult onset and is characterized by a horizontal alveolar bone loss that occurs largely by osteoclastic resorption. Periodontal diseases are induced by bacterial biofilms that stimulate a host response in gingival connective tissue that leads to destruction and loss of bone. ${ }^{7-8} \mathrm{~A}$ critical event in the initiation of periodontal disease is the colonization of teeth by bacteria and bacterial invasion of connective tissue. ${ }^{9}$ Cause-and-effect relationships between periodontal disease and initiating factors have been established by the use of specific inhibitors, which taken together, have indicated that the production of prostaglandins, interleukin (IL)-1, tumor necrosis factor (TNF) and receptor activator of nuclear factor $\kappa \mathrm{B}$ ligand (RANKL) is likely to be an important trigger in the disease process. ${ }^{10-11}$ Osteoprotegerin (OPG) is a member of the TNF receptor family. OPG is a soluble cytokine receptor which binds to OPG ligand (OPGL), also called osteoclast-differentiation factor (ODF). Recombinant OPG increases bone volume in bones from normal and ovariectomized rats. Furthermore, transgenic mice over-expressing

${ }^{1}$ Department of Biochemistry and Molecular Biology, Nihon University School of Dentistry at Matsudo, Matsudo, Japan; ${ }^{2}$ Research Institute of Oral Health, Nihon University

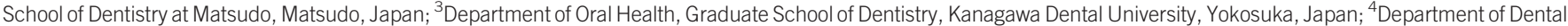

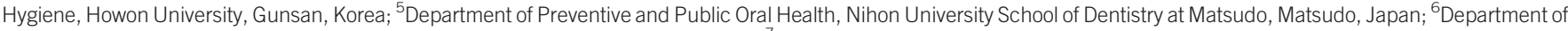

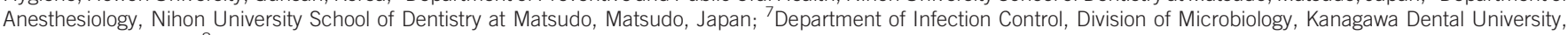
Yokosuka, Japan and ${ }^{8}$ Department of Radiopraxis Science, Kanagawa Dental University, Yokosuka, Japan

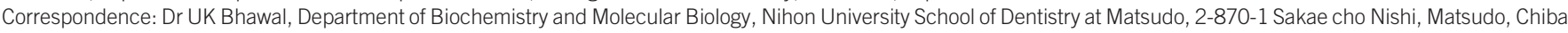
271-8587, Japan

E-mail: bhawal.ujjal.kumar@nihon-u.ac.jp

Accepted 1 April 2015 
OPG have increased bone density compared with their normal littermates. ${ }^{12}$ Recombinant OPG inhibits osteoclast differentiation by binding to OPGL on the cell surface of bone marrow stroma cells and osteoblasts, thereby blocking the binding of OPGL to a putative membrane-bound TNF receptor member on osteoclasts. ${ }^{13}$

RANKL and macrophage colony-stimulating factor (M-CSF) are essential cytokines that induce osteoclast formation. ${ }^{14}$ Recent studies have demonstrated that nuclear factor of activated T cells (NFAT) $\mathrm{cl}$ is a master regulator of RANKL-induced osteoclast formation and is required for osteoclast differentiation in response to RANKL and for NFATc1-deficient embryonic stem cells that fail to differentiate in response to RANKL stimulation. ${ }^{15}$ NFATcl also plays a pivotal role in osteoclast activation, fusion and bone resorption. Matrix metalloproteinase 9 (MMP9) and cathepsin $\mathrm{K}$ are both subject to NFATc1 regulation and can degrade organic ingredients of the bone matrix during bone resorption. ${ }^{16}$

Several different models of periodontal disease have been used to establish bacteria as a critical early initiator. In rat models, placement of a ligature around the teeth causes the formation of a pathogenic biofilm, gingival inflammation, and bone loss. ${ }^{17-18}$ In this study, we used Porphyromonas gingivalis-challenged rats which display profound decreases in alveolar bone area densities. The objectives of the present work were to determine whether fluoride has a regulatory effect on osteoclasts, to observe the influence of $\mathrm{NaF}$ on alveolar bone resorption and to evaluate the cellular and pathological alterations that occur in periodontitis exposed to various NaF concentrations. We demonstrate that fluoride acts as a negative modulator of RANKLinduced osteoclastogenesis via the reduced expression of MMP9 and cathepsin K. Using bone marrow cells isolated from rat femurs as a source of osteoclast progenitors, our data reveal that fluoride not only inhibits osteoclast formation but also attenuates NFATc1 expression during osteoclastogenesis. Our results suggest that fluoride suppresses bone loss, while $P$. gingivalis induces the production of inflammatory factors that stimulate osteoclastic bone resorption.

\section{MATERIALS AND METHODS}

\section{Preparation of bacteria}

P. gingivalis ATCC 33277 was grown in brain heart infusion (BHI) broth supplemented with $5 \mathrm{mg} \cdot \mathrm{mL}^{-1}$ yeast extract, $5 \mu \mathrm{g} \cdot \mathrm{mL}^{-1}$ hemin, and $0.2 \mu \mathrm{g} \cdot \mathrm{mL}^{-1}$ vitamin $\mathrm{K}_{1}$. Bacterial cells were grown under anaerobic conditions $\left(85 \% \mathrm{~N}_{2}, 10 \% \mathrm{H}_{2}\right.$, and $\left.5 \% \mathrm{CO}_{2}\right)$ at $37^{\circ} \mathrm{C}$ for $24 \mathrm{~h}$.

\section{Antibacterial activity against $P$. gingivalis}

Exponentially growing $P$. gingivalis cells were washed and suspended in phosphate-buffered saline (PBS) to an optical density (OD) of 1.0 at $600 \mathrm{~nm}$, which equated to approximately $4 \times 10^{9}$ colony-forming units (CFU) per $\mathrm{mL}$. About $10 \mu \mathrm{L}$ of the bacterial suspension was exposed for 0,10 , and $60 \mathrm{~min}$ to $1 \mathrm{~mL}$ of 5,50 , or $500 \mu \mathrm{mol} \cdot \mathrm{L}^{-1} \mathrm{NaF}$ (Wako Pure Chemical Industries, Tokyo, Japan), or the same volume of PBS as a control. At the end of the incubation period, 10-fold serial dilutions were made in PBS and $100 \mu \mathrm{L}$ of each dilution was spread onto a BHI blood agar plate. The number of CFUs was determined after 7 days incubation in an anaerobic atmosphere. Each experiment was performed three times, and the mean values of experiments are shown. Bactericidal activity was defined as a reduction in viable bacteria of $>3 \log _{10} \mathrm{CFU} \cdot \mathrm{mL}^{-1}$ at any of the incubation periods tested.

\section{Experimental periodontitis}

Eighteen, 3-week-old male Sprague-Dawley rats (CLEA Japan, Tokyo, Japan) were obtained and housed in cages for 2 weeks before starting the experimental period to acclimatize. As shown in Figure 1, the rats were given sulfamethoxazole $\left(1 \mathrm{mg} \cdot \mathrm{mL}^{-1}\right)$ and trimethoprim $\left(200 \mu \mathrm{g} \cdot \mathrm{mL}^{-1}\right)$ in their drinking water for 4 days to reduce any original oral microorganisms, followed by a 3-day antibiotic-free period before starting the oral challenges with bacteria. Rats were divided into the following three groups of 6 rats each. Group A received only 5\% carboxymethyl cellulose (CMC) (control group). Group B was orally challenged with $P$. gingivalis ATCC 33277 ( $P$. g. group). Each rat infected with $P$. gingivalis received $0.5 \mathrm{~mL}\left(1.0 \times 10^{8}\right.$ cells per $\left.\mathrm{mL}\right)$ of the bacterial suspension in $5 \%$ CMC by oral gavage at 8,10 , and 12 days. Group $C$ was treated with $500 \mu \mathrm{mol} \cdot \mathrm{L}^{-1}$ fluoride in their drinking water (P. g. $+\mathrm{NaF}$ group) after the three $P$. gingivalis treatments. All rats were sacrificed 30 days after the final infection and horizontal alveolar bone loss was measured using a morphometric method. The experimental procedures of this study were reviewed and approved by the Committee of Ethics on Animal Experiments of Kanagawa Dental College.

\section{Measurement of alveolar bone resorption}

The left sides of the upper jaws of all rats were used as dry specimens for measuring horizontal alveolar bone loss. The upper jaws were de-fleshed after $10 \mathrm{~min}$ in an autoclave and were then immersed in $3 \%$ hydrogen peroxide, rinsed, air dried, and stained with $1 \%$ methylene blue. Horizontal alveolar bone loss around the maxillary molars

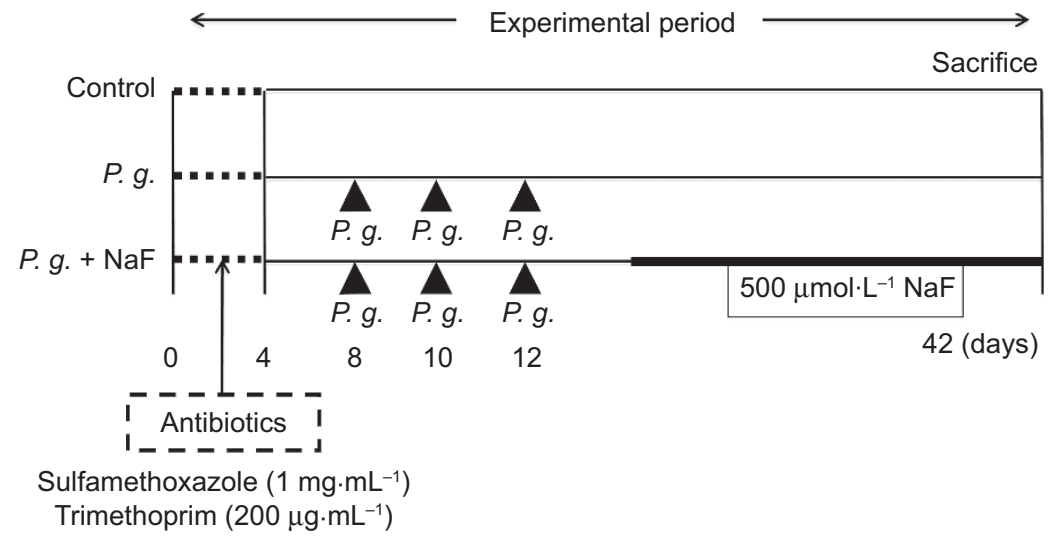

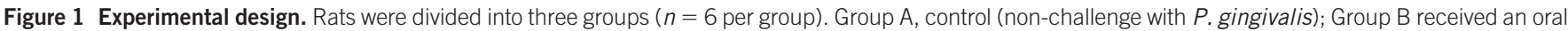

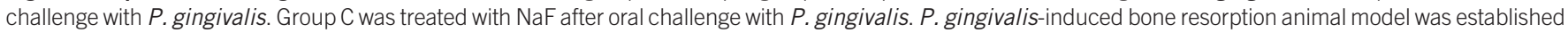

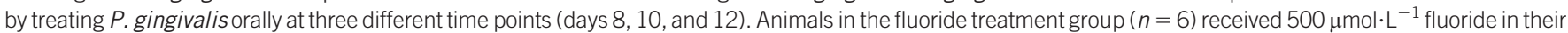
drinking water for 30 days. All rats $(n=18)$ were sacrificed on day 42. P. g., Porphyromonas gingivalis. 
was evaluated morphometrically. In brief, the distance between the cemento-enamel junction (CEJ) and the alveolar bone crest (ABC) was measured at seven buccal sites per rat. Measurements were made under a stereomicroscope $(\times 40$ magnification) fitted with a digital high-definition system (Digital HD microscope VH-7000; KEYENCE, Osaka, Japan), standardized to provide measurements in millimeters.

\section{Three-dimensional architectural parameters and bone morphometric analysis}

For trabecular architectural analysis, three-dimensional imaging data for the mandibular bone were collected using micro-computed tomography (micro-CT) (MCT-CB100MF; Hitachi medico, Tokyo, Japan). The mandibular bone was scanned at the region of $3.4 \mathrm{~mm}$ centered on the first molar teeth. Volume data for 200 consecutive coronary slices were obtained from each sample under the following exposure conditions: tube voltage, $70 \mathrm{kV}$; tube current, $100 \mu \mathrm{A}$; voxel size, $17 \mu \mathrm{m} \times$ $17 \mu \mathrm{m} \times 17 \mu \mathrm{m}$. TRI 3D-BON software (Ratoc System Engineering, Tokyo, Japan) was used to measure the trabecular architecture. The 3D trabecular structure parameters, namely, bone volume per tissue volume (BV/TV), bone surface per bone volume (BS/BV), trabecular number (Tb.N), trabecular thickness (Tb.Th), and trabecular separation (Tb.Sp) were analyzed in the 3D-reconstructed images. Differences in mean values between the control and experimental groups were calculated for each parameter to obtain mean percentage changes.

\section{Tartrate-resistant acid phosphatase staining and immunohistochemistry}

The right sides of the upper jaws were not autoclaved and were used for histological study. They were fixed with $4 \%$ paraformaldehyde $(\mathrm{pH}$ 7.4) overnight and then rinsed with $0.1 \mathrm{~mol} \cdot \mathrm{L}^{-1} \mathrm{PBS}$. After decalcification in $15 \%$ ethylenediaminetetraacetic acid for approximately 6 weeks at $4{ }^{\circ} \mathrm{C}$, the samples were rinsed again with $0.1 \mathrm{~mol} \cdot \mathrm{L}^{-1} \mathrm{PBS}$ and embedded in paraffin. The paraffin sections were cut at a thickness of $4 \mu \mathrm{m}$ in the mesial-distal direction and were stained with tartrateresistant acid phosphatase (TRAP; TRAP Staining Kit; Wako, Tokyo, Japan). TRAP staining was performed in accordance with the manufacturer's instructions. Red-stained multinucleated (TRAP-positive) cells were defined as osteoclasts and were examined under an optical microscope ( $\times 200$ magnification) (Nikon, Tokyo, Japan).

Formalin-fixed, paraffin-embedded specimens were subjected to antigen retrieval, blocking of endogenous peroxidase and were then rinsed with PBS. Immunostaining was performed using a CSA system (DAKO, Carpinteria, CA, USA). Sections were initially immersed in target retrieval solution (DAKO, Carpinteria, CA, USA) at $97{ }^{\circ} \mathrm{C}$ for $40 \mathrm{~min}$, and subsequent steps were performed according to the instructions of the manufacturer. Endogenous peroxidase activity was blocked with REAL peroxidase-blocking solution (S2023, DAKO, Carpinteria, CA, USA) for $30 \mathrm{~min}$. Antibodies against cathepsin $\mathrm{K}$ (1:80; LifeSpan Biosciences, Seattle, WA, USA), NFATc1 (1:100; Abcam, Tokyo, Japan), MMP9 (1:100; Cell Signaling Technology, Danvers, MA, USA) and IL-1 $\beta$ (1:50; Santa Cruz Biotechnology, Santa Cruz, CA, USA), were used as primary antibodies and were incubated overnight at $4{ }^{\circ} \mathrm{C}$. The secondary antibodies were incubated at room temperature for $30 \mathrm{~min}$. The immunostaining of all specimens was performed simultaneously to ensure the same antibody reaction and 3,3'-diaminobenzidine (DAB) exposure conditions. In sections stained with antibodies, the area with the highest fraction of antibodystained cells was chosen using a $\times 10$ objective magnification. After that, a $\times 40$ objective was used to count arbitrarily the fraction of positive cells among 200 cells.

\section{Enzyme-linked immunosorbent assay}

Human gingival fibroblasts (HGFs) were prepared from healthy gingival tissues from donors $(n=3)$ with their informed consent and Ethics Committee approval. HGFs were plated in $35-\mathrm{mm}$ culture dishes (Corning Corning, NY, USA) containing Dulbecco's modified Eagle's medium (DMEM; Sigma, St Louis, MO, USA) supplemented with $10 \%$ fetal bovine serum (FBS) (HyClone, South Logan, UT, USA), $100 \mathrm{u} \cdot \mathrm{mL}^{-1}$ penicillin, $100 \mu \mathrm{g} \cdot \mathrm{mL}^{-1}$ streptomycin and $1 \mu \mathrm{g} \cdot \mathrm{mL}^{-1}$ amphotericin B. When the cells formed a sub-confluent monolayer, they were harvested and seeded in 100-mm culture dishes (Corning, Corning, NY, USA). HGFs $\left(2 \times 10^{5}\right.$ cells per well in 6 -well plates $)$ at the fourth passage were used in the experiments. Human recombinant IL-1 $\beta$ $\left(10 \mathrm{ng} \cdot \mathrm{mL}^{-1}\right.$ ) was added to the cells for $24 \mathrm{~h}$. Cells were treated with 5 or $500 \mu \mathrm{mol} \cdot \mathrm{L}^{-1} \mathrm{NaF}$ for $1 \mathrm{~h}$ prior to treatment with IL- $1 \beta$. enzymelinked immunosorbent assay (ELISA) was performed to measure IL-6 and IL-8 concentrations in cell culture media of HGFs according to the instructions of the manufacturer (Millipore Billerica, MA, USA) in triplicate. The experiment was repeated twice.

\section{Osteoclast formation}

Femurs were aseptically removed from 4- to 6-week old SpragueDawley rats (CLEA Japan, Tokyo, Japan), and bone marrow cells were flushed out with a sterile 21-gauge syringe. The cells were cultured in $\alpha$-MEM (HyClone Laboratories, Waltham, MA, USA) containing $10 \%$ FBS with M-CSF $\left(30 \mathrm{ng} \cdot \mathrm{mL}^{-1}\right.$ ) for 3 days. Floating cells were removed and adherent cells were used as osteoclast precursors. To generate osteoclasts, cells were cultured with $\mathrm{M}-\mathrm{CSF}\left(30 \mathrm{ng} \cdot \mathrm{mL}^{-1}\right)$ and RANKL $\left(100 \mathrm{ng} \cdot \mathrm{mL}^{-1}\right)$ for 7 days. The cells were then cultured with the indicated concentrations of fluoride for 3 days.

\section{Quantitative real-time reverse transcriptase-polymerase chain reaction}

Total RNA was isolated from cultured bone marrow cells using an RNeasy Mini kit (Qiagen, Hilden, Germany). First-strand cDNA was synthesized from $1 \mathrm{mg}$ total RNA using High Capacity RNA-to-cDNA Master Mix (Applied Biosystems, Carlsbad, CA, USA). A one-hundredth aliquot of each cDNA was subjected to real-time reverse transcriptase-polymerase chain reaction (RT-PCR) using TaqMan Gene Expression Assays (Applied Biosystems, Carlsbad, CA, USA) for OPG, RANKL, NFATc1, and cathepsin K and Pre-Developed TaqMan Assay Reagents (Applied Biosystems, Carlsbad, CA, USA) for $\beta$-actin (ACTB) as an internal control. Three independent measurements were averaged and relative gene expression levels were calculated as a ratio to ACTB expression of each cell.

\section{Western Blotting}

Cells were lysed in RIPA lysis buffer (Santa Cruz Biotechnology, Santa Cruz, CA, USA). Protein concentrations were determined using a BCA Protein Assay Kit (Pierce Biotechnology, Rockford, IL, USA). sodium dodecylsulphate (SDS)-polyacrylamide gel electrophoresis (PAGE) was calibrated with molecular weight markers (Bio-Rad, Hercules, CA, USA). NFATc1 (1:500; Abcam, Tokyo, Japan), and glyceraldehyde phosphate dehydrogenase (GAPDH; 1:1 000; Cell Signaling Technology, Danvers, MA, USA) were used as primary antibodies. Anti-mouse and anti-rabbit secondary antibodies (Cell Signaling Technology, Danvers, MA, USA) were each used at a dilution of 1:2 000. Bound antibodies were visualized by chemiluminescence using the ECL Plus Western Blotting Detection System (Amersham, Uppsala, Sweden), and images were analyzed by a Luminescent Image Analyzer (LAS-3000; Fuji Film, Tokyo, Japan). 


\section{Statistical analysis}

Significant differences were analyzed by Fisher's exact test and Dunnett's test. Data analysis was also performed using SPSS (Statistical Package for the Social Sciences 18.0 SPSS Chicago, IL, USA). Differences between the positively stained cells in the three groups were tested by parametric Kruskal-Wallis and Mann-Whitney as a post-hoc procedure. A $P$-value of less than 0.01 is considered statistically significant.

\section{RESULTS}

Antibacterial activity of $\mathrm{NaF}$ against $P$. gingivalis

Treatment of $P$. gingivalis with $\mathrm{NaF}$ at various concentrations reduced the number of viable cells over the experimental period in a concentration-dependent manner (Figure 2). Bactericidal activity against $P$. gingivalis was observed for the 60 -min treatment and the number of CFU of $P$. gingivalis decreased from $(4.21 \pm 0.12) \times 10^{7}$ to $(1.33 \pm$ $0.25) \times 10^{4}$ per $\mathrm{mL}$ (Figure 2 ).

\section{Measurement of alveolar bone resorption}

In order to evaluate bacteria-induced bone resorption, the right sides of the upper jaws were prepared 30 days following the last infection with $P$. gingivalis. The level of bone loss was evaluated by the distance from $\mathrm{CEJ}$ to $\mathrm{ABC}$ at the buccal area on the left side of the maxilla (Figure 3a). The level of bone loss the P. g. group was significantly increased compared to the control and the fluoride-treated groups $(P<0.05)$ (Figure 3b).

Fluoride reduces bone destruction in experimental periodontitis When the rats were treated with fluoride, there was a reduction in the level of inflammation. TRAP staining showed more active osteoclasts

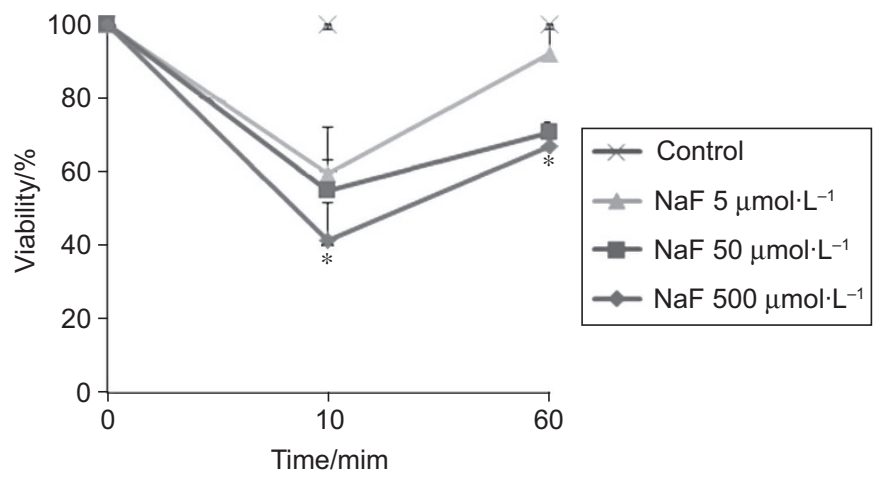

Figure 2 Antibacterial effect of $\mathrm{NaF}$ on P. gingivalis. Bacterial cells were treated with various concentrations of $\mathrm{NaF}$ or PBS for the indicated time. Each experiment was carried out three times, and the results are shown as mean values of the experiments. Bactericidal activity was defined as a reduction in viable bacteria of $\mathrm{CFU} \cdot \mathrm{mL}^{-1}$ at any of the incubation periods tested. $* P<0.05$. PBS, phosphatebuffered saline.
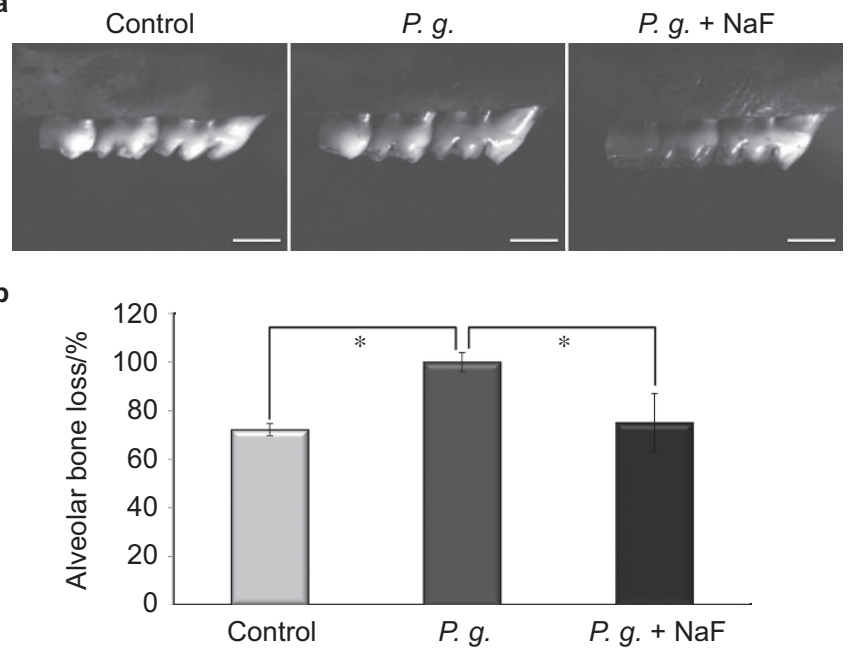

Figure 3 Alveolar bone loss induced by bacterial infection. (a) The distance between the cemento-enamel junction (CEJ) and the alveolar bone crest (ABC) was evaluated for horizontal alveolar bone loss on the left side of maxilla. Scale bar: $200 \mu \mathrm{m}$. (b) These results represent the means of data obtained from six rats and are expressed as means \pm standard deviation. ${ }^{*} P<0.05$. P. g., Porphyromonas gingivalis.

in the periodontal tissue from the $P . g$. group than from the control group, and fluoride treatment decreased osteoclast formation in the periodontal tissue (Figure 4a, Table 1). Cathepsin K protein expression appeared highest in osteoclasts adjacent to the alveolar bone in the P. g. group (Figure 4a, Table 1). Immunohistochemical analysis revealed the increased expression of NFATc1, MMP9, and IL-1 $\beta$ in the $P . g$. group, while fluoride treatment decreased their expression (Figure 4b, Table 1).

\section{Micro-CT analysis}

Micro-CT analysis demonstrated that $P$. gingivalis caused a decrease in bone area compared with the control group (Figure 5). When the data are expressed in terms of bone loss, treatment with fluoride reduced bone loss compared with the $P$. g. group. Treatment with vehicle alone did not significantly change the bone volume. Trabecular bone thickness was significantly different between the P. g. group and the P. g. $+\mathrm{NaF}$ group. Fluoride application was associated with increased BV/BV and decreased Tb. Sp. A trend of decreased BS/BV was observed in the control and the P.g. $+\mathrm{NaF}$ groups compared to the P. g. group (Table 2).

\section{Anti-inflammatory effect of $\mathrm{NaF}$}

To determine whether NaF-treated IL- $1 \beta$-exposed cells acquired antiinflammatory activity, we performed ELISA with conditioned media

Table 1 Values in the tissue specimens with P. g. and P. g. + NaF and control groups

\begin{tabular}{|c|c|c|c|c|c|}
\hline Groups* & TRAP & Cathepsin K & NFATc1 & MMP9 & $\mathrm{IL}-1 \beta$ \\
\hline Control & $1.8 \pm 0.45^{a}$ & $1.2 \pm 0.45^{a}$ & $3.0 \pm 0.71^{a}$ & $1.2 \pm 0.45^{a}$ & $3.6 \pm 0.89^{a}$ \\
\hline P.g. & $16.6 \pm 2.07^{b}$ & $16.4 \pm 1.52^{b}$ & $16.8 \pm 1.79^{b}$ & $12.8 \pm 1.30^{\mathrm{b}}$ & $16.0 \pm 1.00^{\mathrm{b}}$ \\
\hline P. g. + NaF & $8.4 \pm 1.14^{c}$ & $10.8 \pm 1.64^{c}$ & $8.6 \pm 0.89^{c}$ & $3.6 \pm 1.34^{c}$ & $9.6 \pm 1.14^{c}$ \\
\hline
\end{tabular}

All values are mean \pm standard deviation.

IL, interleukin; MMP, matrix metalloproteinase; NFAT, nuclear factor of activated T cells; P. g., Porphyromonas gingivalis; TRAP, tartrate-resistant acid phosphatase.

$* P<0.01$ by Kruskal-Wallis test.

a,b,c The letter indicates significant difference by Mann-Whitney post-hoc test $(P<0.01)$. 

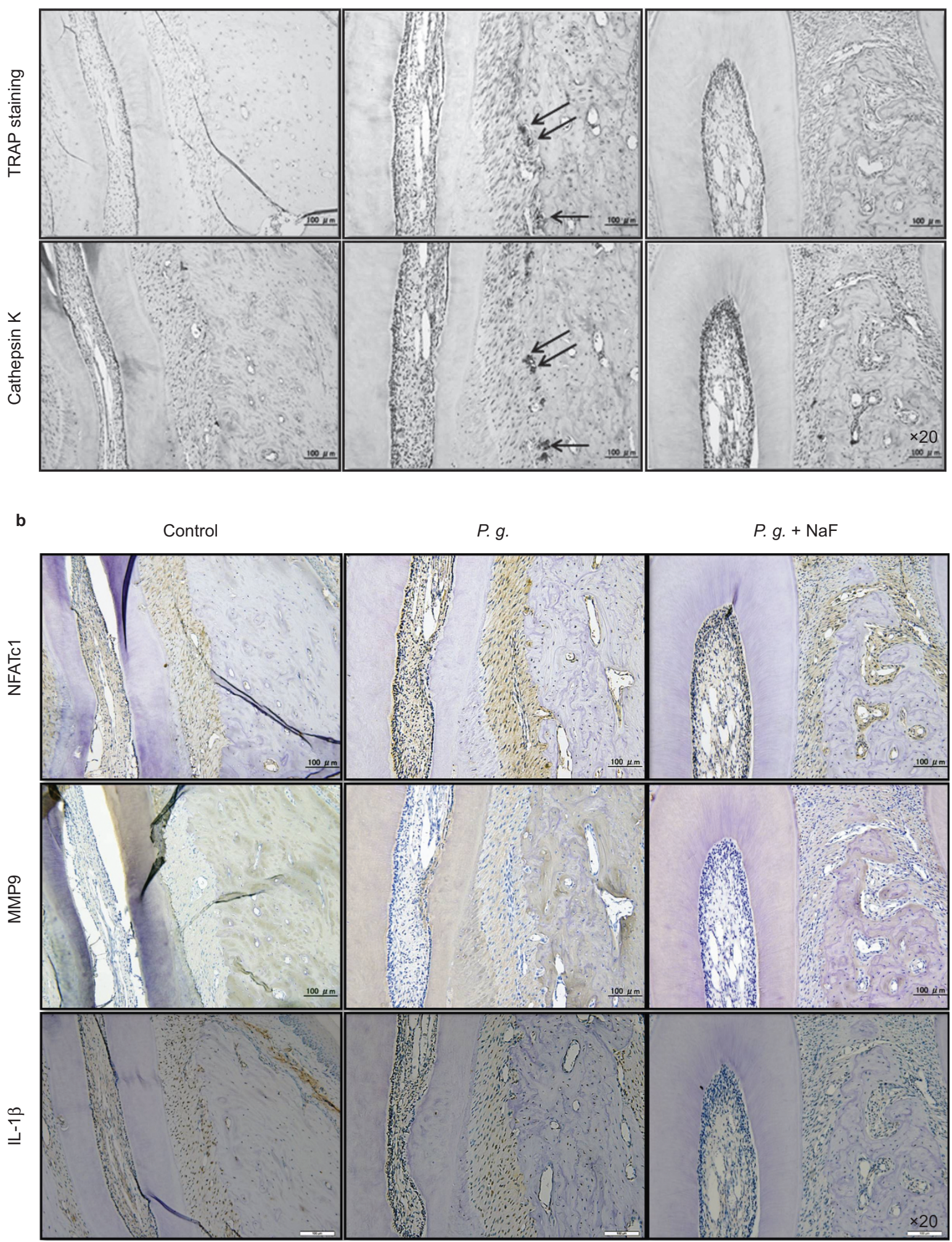

Figure 4 Fluoride decreases osteoclast formation in periodontal tissues. (a) TRAP staining of the upper maxilla. The arrows showing TRAP-positive cells were defined as osteoclasts under a microscope at $\times 200$ magnification. Immunohistochemical analysis revealed cathepsin K protein expression in the TRAP-positive cells in the $P$. g. group. Scale bars show $100 \mu \mathrm{m}$. (b) Increased expression of NFATc1, MMP9, and IL-1 $\beta$ was observed in the $P$. g. group, while fluoride treatment decreased their expression. IL, interleukin; MMP, matrix metalloproteinase; NFAT, nuclear factor of activated T cells; P. g., Porphyromonas gingivalis; TRAP, tartrate-resistant acid phosphatase. 


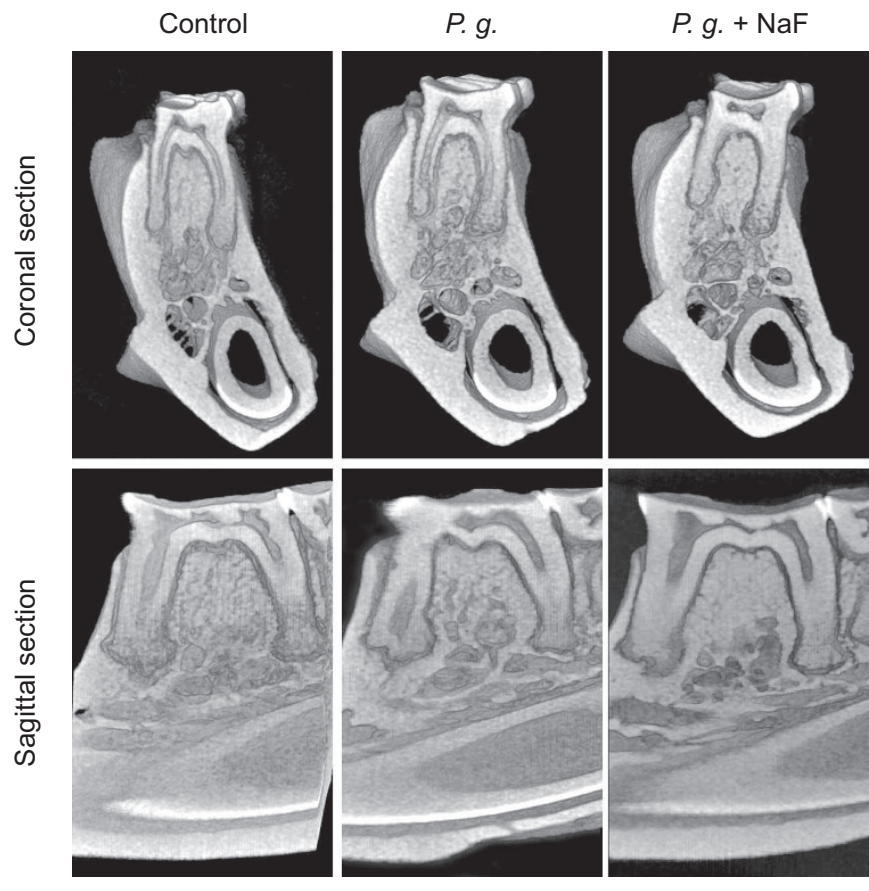

Figure 5 The inhibitory effect of fluoride on $\boldsymbol{P}$. gingivalis-induced bone absorption. After acclimating to the environment of the Animal Center and antibiotic treatment, 6-week-old Sprague-Dawley rats were orally challenged at 3 time points with $P$. gingivalis ATCC 33277 or with vehicle. The $P$. g. + NaF group received $500 \mu \mathrm{mol} \cdot \mathrm{L}^{-1}$ fluoride in the drinking water for the next 30 days, as shown in Figure $1(n=6)$. Coronal and sagittal reconstructed 3D images of the mandibular molar regions of each group were obtained with a $\mu \mathrm{CT}$ apparatus. P. g., Porphyromonas gingivalis; $\mu \mathrm{CT}$, micro-computed tomography.

from HGFs cultured with human recombinant IL-1 $\beta$ and 5 or $500 \mu \mathrm{mol} \cdot \mathrm{L}^{-1} \mathrm{NaF}$. As shown in Figure $6,500 \mu \mathrm{mol} \cdot \mathrm{L}^{-1} \mathrm{NaF}$ significantly decreased the IL- $1 \beta$-induced IL- 6 and IL- 8 production in HGF cells.

Effect of fluoride on the RANKL-associated signaling pathway We used various concentrations of fluoride and investigated its potential to reduce osteoclast formation and to suppress the RANKL-associated signaling pathway. Given that fluoride disturbed the expression of NFATc1, we next focused on osteoclastic differentiation, and more particularly on the induction of NFATcl gene

Table 2 The inhibitory effect of fluoride on $P$. gingivalis-induced bone absorption

\begin{tabular}{lccc}
\hline Parameters & Control & P.g. & P.g. $+\mathrm{NaF}$ \\
\hline$(\mathrm{BV} / \mathrm{TV}) / \%$ & $55.6 \pm 5.2$ & $46.8 \pm 3.3^{\mathrm{a}, \mathrm{b}}$ & $54.8 \pm 3.1$ \\
$(\mathrm{BS} / \mathrm{BV}) / \%$ & $15.7 \pm 0.9$ & $19.2 \pm 3.4$ & $16.8 \pm 0.2$ \\
$\mathrm{~Tb} . \mathrm{Th} /\left(\mu \mathrm{mol} \cdot \mathrm{L}^{-1}\right)$ & $127.9 \pm 7.4$ & $106.6 \pm 10.1^{\mathrm{a}, \mathrm{b}}$ & $119.3 \pm 1.8$ \\
$\mathrm{~Tb} . \mathrm{Sp} /\left(\mu \mathrm{mol} \cdot \mathrm{L}^{-1}\right)$ & $103.1 \pm 18.7$ & $121.9 \pm 3.4$ & $106.6 \pm 10.8$ \\
\hline
\end{tabular}

BV/TV, bone volume/tissue volume; BS/BV, bone surface/bone volume; $P$. g., Porphyromonas gingivalis; Tb. Th: trabecular thickness; Tb. Sp: trabecular separation.

Data representing BV/TV compared to the control group for the microstructural indices of trabecular bone density. Tb. Th was also measured using micro-computed tomography scanner software. The results are expressed as means \pm standard deviation.

${ }^{\mathrm{a}} P<0.05$ vs. control; ${ }^{\mathrm{b}} P<0.05$ vs. $P$. g. $+\mathrm{NaF}$. Statistical analyses were performed using two-way analysis of variance followed by Dunnett's test.

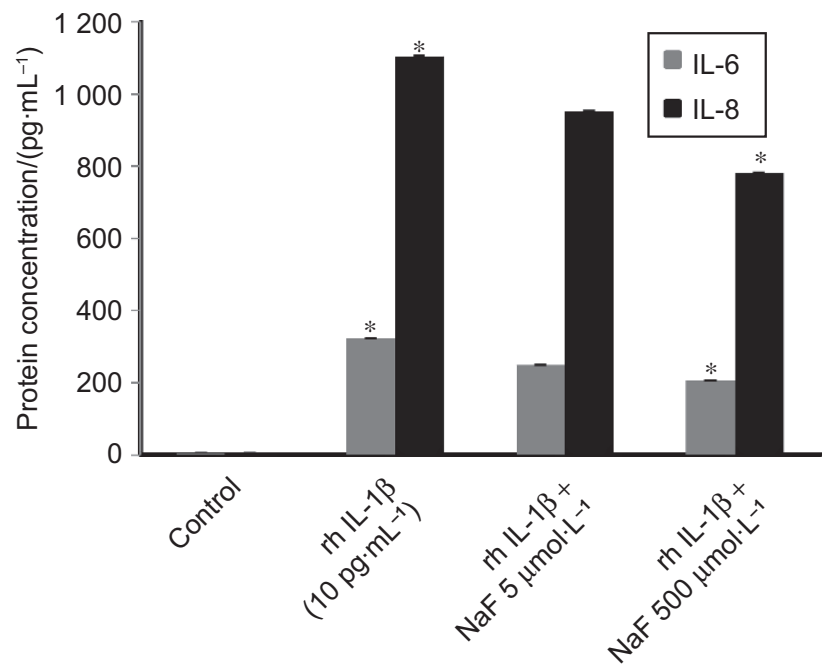

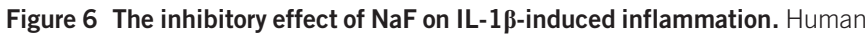
primary gingival fibroblasts $\left(2 \times 10^{5}\right)$ were treated with $10 \mathrm{ng} \cdot \mathrm{mL}^{-1}$ human recombinant IL-1 $\beta$ for $24 \mathrm{~h}$ with or without $\mathrm{NaF}$, and levels of IL-6 and IL-8 in the culture supernatants were measured by ELISA. Pretreatment with $500 \mu \mathrm{mol} \cdot \mathrm{L}^{-1} \mathrm{NaF}$ significantly decreased IL- 6 and IL-8 production in HGFs. The experiments were repeated twice in triplicate. rhIL, recombinant human interleukin; ELISA, enzyme-linked immunosorbent assay; HGF, Human gingival fibroblast.

expression, that leads to NFATc1 protein level up-regulation. Cells were cultured with M-CSF $\left(30 \mathrm{ng} \cdot \mathrm{mL}^{-1}\right)$ and RANKL $\left(100 \mathrm{ng} \cdot \mathrm{mL}^{-1}\right)$ for 7 days to stimulate osteoclastogenesis. We measured NFATc1 gene expression after a 72-h incubation period of cells with both RANKL and fluoride. As shown in Figure 7, fluoride induced a down-regulation of NFATc1 gene expression, and this

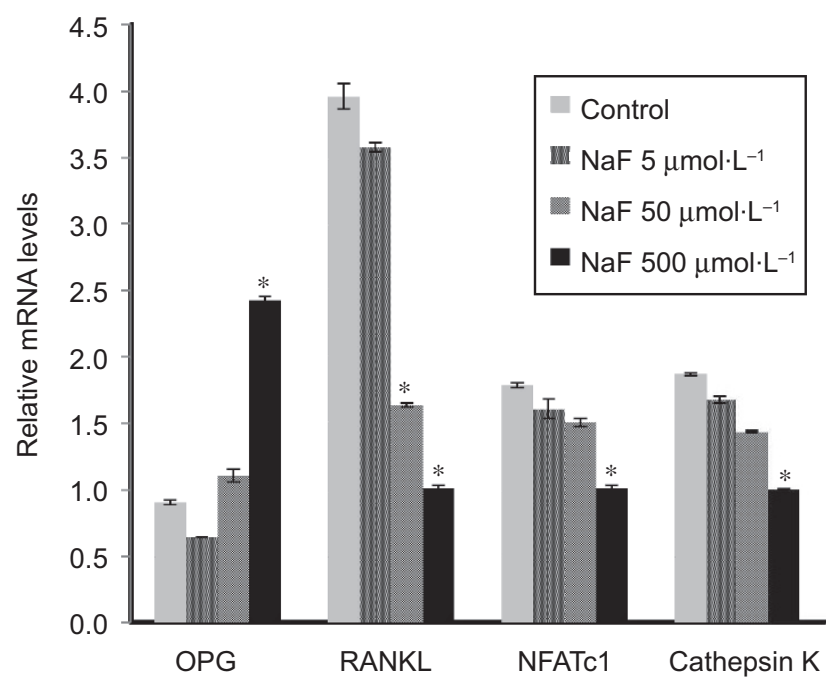

Figure 7 Effect of $\mathrm{NaF}$ on the suppression of osteoclast differentiation. Bone marrow cells were cultured with M-CSF $\left(30 \mathrm{ng} \cdot \mathrm{mL}^{-1}\right)$ and RANKL $\left(100 \mathrm{ng} \cdot \mathrm{mL}^{-1}\right)$ for 7 days to stimulate osteoclastogenesis. The cells were then cultured with the indicated concentrations of fluoride for 3 days. mRNA expression levels of genes were determined by quantitative Real-time RT-PCR and compared with $\beta$-actin as a control. The results are expressed as means \pm standard deviation. $n=3$ per group $(* P<0.05)$. NFAT, nuclear factor of activated T cell; OPG, Osteoprotegerin; RANKL, receptor activator of nuclear factor $\kappa B$ ligand; RT-PCR, reverse transcriptase-polymerase chain reaction. 


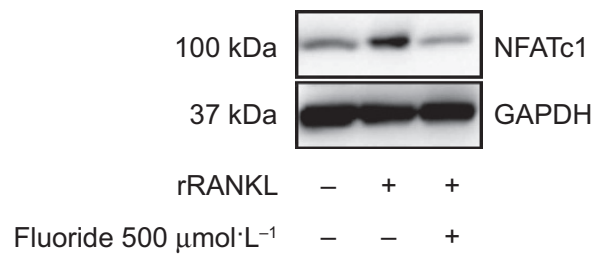

Figure 8 Fluoride disturbs the expression of NFATc 1 protein. The cell extracts were analyzed by immunoblotting with specific antibodies for NFATC1 and GAPDH. An equal amount of protein was loaded in each lane, as demonstrated by the level of GAPDH. Bound antibodies were visualized by chemiluminescence using the ECL Plus Western Blotting Detection System, and images were analyzed by a Luminescent Image Analyzer. The experiment was repeated three times. GAPDH, glyceraldehyde phosphate dehydrogenase; NFAT, nuclear factor of activated T cell; rRANKL, recombinant receptor activator of nuclear factor $\mathrm{\kappa B}$ ligand.

inhibition was significant when $500 \mu \mathrm{mol} \cdot \mathrm{L}^{-1}$ fluoride was used in rat osteoclasts. Similar results were obtained for the expression of cathepsin K. To correlate the effects of fluoride at the transcript level with NFATc1 protein content, we performed immunolabeling of rat osteoclast cells treated with RANKL/fluoride for $72 \mathrm{~h}$. In the absence of RANKL, we detected a weak expression of NFATc1, while treatment with RANKL induced a strong expression of NFATc1 protein. Lastly, we observed that treatment with $500 \mu \mathrm{mol} \cdot \mathrm{L}^{-1}$ fluoride prevented RANKL-induced NFATc1 protein expression (Figure 8).

\section{DISCUSSION}

Previous studies primarily focused on the effect of fluoride on osteoblast activity where it was shown to have a positive effect on the differentiation of osteoblasts, bone nodule formation and bone regeneration. However, the effect of fluoride on osteoclast function in bone-lytic diseases, such as periodontitis, is poorly documented. Data from the current study using rat bone marrow cells in culture and a $P$. gingivalisinduced periodontitis model suggest that osteoclast precursors are sensitive to low levels of fluoride. The fluoride concentrations in our experiments were chosen according to a previous study that resulted in measurable effects on cultured cells. ${ }^{19}$ Fluoride can inhibit the function of isolated osteoclasts at 0.5 to $1.0 \mathrm{mmol} \cdot \mathrm{L}^{-1}$ in vitro. ${ }^{20}$ Fluoridated drinking water markedly inhibited the bone resorption induced by hydrocortisone $\mathrm{e}^{21}$ and showed a lower histologic incidence of alveolar bone resorption after the injection of parathormone. ${ }^{22}$

In previous studies, researchers found that the number of osteoclasts was proportional to the capacity of bone resorption. ${ }^{20,23}$ The inhibition of bone resorption by fluoride in a variety of model systems ${ }^{24-25}$ suggests that a high intake of fluoride might protect against alveolar bone loss in periodontal disease. Human studies of periodontal disease in relation to the intake of fluoride have yielded equivocal results, although they have not focused specifically on alveolar bone loss. ${ }^{26}$ Okuda et al. ${ }^{20}$ suggested that $500 \mu \mathrm{mol} \cdot \mathrm{L}^{-1} \mathrm{NaF}$ is the optimal concentration that may be encountered by osteoclasts or osteoblasts in vivo, and fluoride can inhibit the function of isolated osteoclasts at $500 \mu \mathrm{mol} \cdot \mathrm{L}^{-1}$ to $1.0 \mathrm{mmol} \cdot \mathrm{L}^{-1}$ or nearphysiologic concentrations around $800 \mu \mathrm{mol} \cdot \mathrm{L}^{-1} \cdot{ }^{27}$ However, the regulatory molecular mechanism of fluoride on osteoclast function has not been well clarified. In the present study, $\mathrm{NaF}$ exerts an antibacterial effect against the potential growth of oral anaerobe $P$. gingivalis. NaF can inhibit the initial adhesion and the colonization of $P$. gingivalis, which involves surface antibacterial activity. Fluorine ions released from fluoride can affect bacterial metabolism as an enzyme inhibitor. $\mathrm{NaF}$ also displayed inhibitory effects on $P$. gingivalis-induced osteoclastogenesis. In the bone marrow cell culture system where osteoclast formation is governed by soluble RANKL and M-CSF, we observed an even more potent inhibitory effect of $\mathrm{NaF}$ on osteoclast formation. These observations indicate that the anti-osteoclastogenic activity of $\mathrm{NaF}$ is on the precursor cells in osteoclast differentiation. Taken together, we suggest that the application of fluoride has a potential synergistic effect in maintaining a healthy oral microbial equilibrium and thus represents a promising ecological approach to periodontitis management.

Fluoride acts on protease activity in the extracellular matrix and inhibits matrix degradation. ${ }^{28}$ During osteoclast-mediated bone resorption, the organic constituents of bone are degraded by lysosomal enzymes, which are secreted directionally into the resorbing compartment. $^{29}$ A novel cysteine protease, cathepsin $\mathrm{K}$, appears to be selective for osteoclasts and plays a pivotal role in bone matrix degradation. $^{30-31}$ Sections of $P$. gingivalis-induced periodontitis showed intense expression of cathepsin K protein. Our data demonstrate that cathepsin $\mathrm{K}$ is correlated not only with fluoride exposure but also with the changes of bone resorption markers.

Osteoclast bone resorption is a complex process involving many functional genes. NFATc1 plays a key role in this process by regulating osteoclast fusion and bone resorption activity. MMP9 and cathepsin K are both subject to NFATc1 regulation and can degrade the organic ingredients of bone matrix during bone resorption. ${ }^{16,32}$ In this study, we found that NFATc1 expression is significantly decreased in the fluoride treatment group compared with the control group. These results suggest that the molecular mechanisms in bone resorption are reduced by fluoride through the inhibition of NFATc1 and downstream functional genes, including MMP9 and cathepsin K, thus leading to an impaired capacity of osteoclast acidification, reduced secretion of proteolytic enzymes, and decreased bone resorption.

There are several limitations of our study. First, the age of the animals studied: Periodontal disease in humans has an adult onset. We used a young rat $P$. gingivalis-challenged periodontitis model that provided large amounts of favorable data. However, it is difficult to determine the mechanism of accurate alveolar bone loss induced by the challenge of $P$. gingivalis in adult rats because of their natural periodontitis. Unlike the chronic process in human periodontitis, which takes many years, in adult rats the disease progresses to a chronic destruction of soft and hard tissues within a few weeks. Second, the viability of cells in the presence of NaF: In our study, the viability of bone marrow cells was not significantly influenced by $\mathrm{NaF}$ at the $\mu \mathrm{mol} \cdot \mathrm{L}^{-1}$ level, whereas it was substantially reduced by $\mathrm{NaF}$ at the $\mathrm{mM}$ level (data not shown). Oguro et al. ${ }^{33}$ also supported this notion. They hypothesized that membrane-bound antigens and cytokines could be accumulated and apparently up-regulated in cells that have survived in the presence of $500 \mu \mathrm{mol} \cdot \mathrm{L}^{-1} \mathrm{NaF}$. Future studies should attempt to further confirm this hypothesis. Third, the mixed population of bone marrow cells: The in vitro cell culture experiments did not always reflect the in vivo situation because tissue vascularization and the effect of the microenvironment are not accounted for in vitro. In our study, the bone marrow cells of the $\mathrm{NaF}$ group expressed higher levels of OPG than did the control group. We speculate that there is a mixed population of cells, including a small population of mesenchymal stem cells (MSCs), in our culture system. Recently, Oshita et al. ${ }^{34}$ demonstrated that human MSCs express OPG and inhibit osteoclast formation in vitro. It may be that the inhibitory factor OPG secreted from MSCs in the presence of $\mathrm{NaF}$ suppresses osteoclastogenesis in the cellular mix in our bone marrow culture system. We predict the presence of some putative potent inhibitory molecules on the surface of MSCs. Further studies are required to elucidate such regulators in co-culture experiments of MSCs and osteoclasts. 
In summary, our findings clearly show that fluoride has an antiosteoclastogenic potential by reducing the RANKL induction of NFATc1 in osteoclast precursors. Moreover, fluoride prevents the $P$. gingivalis-induced alveolar bone destruction. Thus, our findings indicate that fluoride deserves new evaluation as a potential treatment option in periodontitis associated with excessive osteoclast formation and alveolar bone destruction.

1 Bellows CG, Heersche JN, Aubin JE. The effects of fluoride on osteoblast progenitors in vitro. J Bone Miner Res 1990; 5(Suppl 1): 101-105.

2 Boivin G, Chavassieux P, Chapuy MC et al. Skeletal fluorosis: histomorphometric analysis of bone changes and bone fluoride content in 29 patients. Bone 1989; 10(2): 89-99.

3 Turner $\mathrm{CH}$, Garetto LP, Dunipace AJ et al. Fluoride treatment increased serum IGF-1, bone turnover, and bone mass, but not bone strength in rabbits. Calcif Tissue Int 1997; 61(1): 77-83.

4 Rich C, Ensinck J. Effect of sodium fluoride on calcium metabolism in human beings. Nature 1961; 191: 184-185.

5 Farley JR, Tarbaux N, Hall S et al. Evidence that fluoride-stimulated 3[H]-thymidine incorporation in embryonic chick calvarial cell cultures is dependent on the presence of a bone cell mitogen, sensitive to changes in the phosphate concentration, and modulated by systemic skeletal effectors. Metabolism 1988; 37(10): 988-995.

6 Wergedal JE, Lau KH, Baylink DJ. Fluoride and bovine bone extract influence cell proliferation and phosphatase activities in human bone cell cultures. Clin Orthop Relat Res 1988; 233: 274-282.

7 Graves DT, Cochran D. The contribution of interleukin-1 and tumor necrosis factor to periodontal tissue destruction. J Periodontol 2003; 74(3): 391-401.

8 Haffajee AD, Socransky SS. Introduction to microbial aspects of periodontal biofilm communities, development and treatment. Periodontol 2000 2006; 42: 7-12.

9 Holt SC, Ebersole J, Felton J et al. Implantation of bacteroides gingivalis in non-human primates initiates progression of periodontitis. Science 1988; 239(4835): 55-57.

10 Assuma R, Oates T, Cochran D et al. IL-1 and TNF antagonists inhibit the inflammatory response and bone loss in experimental periodontitis. J Immunol 1998; 160(1): 403-409.

11 Teng YT, Nguyen H, Gao X et al. Functional human T-cell immunity and osteoprotegerin ligand control alveolar bone destruction in periodontal infection. J Clin Invest 2000; 106(6): 59-67.

12 Simonet WS, Lacey DL, Dunstan CR et al. Osteoprotegerin: a novel secreted protein involved in the regulation of bone density. Cell 1997; 89(2): 309-319.

13 Yasuda $\mathrm{H}$, Shima N, Nakagawa $\mathrm{N}$ et al. Osteoclast differentiation factor is a ligand for osteoprotegerin/osteoclastogenesis inhibitory factor and is identical to TRANCE/ RANKL. Proc Natl Acad Sci USA 1998; 95(7): 3597-3602.

14 Boyle WJ, Simonet WS, Lacey DL. Osteoclast differentiation and activation. Nature 2003; 423(6937): 337-342.

15 Takayanagi H, Kim S, Koga T et al. Induction and activation of the transcription factor NFATc1 (NFAT2) integrate RANKL signaling in terminal differentiation of osteoclasts. Dev Cell 2002; 3(6): 889-901.

16 Song I, Kim JH, Kim K et al. Regulatory mechanism of NFATc1 in RANKL-induced osteoclast activation. FEBS Lett 2009; 583(14): 2435-2440.

17 Kenworthy R, Baverel M. Studies of a periodontal tissue lesion in the rat, untreated or treated with chlorhexidine digluconate. J Clin Periodontol 1981; 8(4): 349-358.
18 Liu R, Bal HS, Desta T et al. Diabetes enhances periodontal bone loss through enhanced resorption and diminished bone formation. J Dent Res 2006; 85(6): 510-514.

19 Arakawa Y, Bhawal UK, Ikoma T et al. Low concentration fluoride stimulates cell motility of epithelial cells in vitro. Biomed Res 2009; 30(5): 271-277.

20 Okuda A, Kanehisa J, Heersche JN. The effects of sodium fluoride on the resorptive activity of isolated osteoclasts. J Bone Miner Res 1990; 5(Suppl 1): S115-S120.

21 Zipkin I, Bernick S, Menczel J. A morphological study of the effect of fluoride on the periodontium of the hydrocortisone-treated rat. Periodontics 1965; 3: 111-114.

22 Levy BM, Dreizen S, Bernick S et al. Studies on the biology of the periodontium of marmosets: IX. Effect of parathyroid hormone on the alveolar bone of marmosets pretreated with fluoridated and non-fluoridated drinking water. J Dent Res 1970; 49(4): 816-821.

23 Debinski A, Nowicka G. Effect of sodium fluoride on ectopic induction of bone tissue. Ann Acad Med Stetin 2004; 50(Suppl 1): 23-27.

24 Gedalia I, Hodge HC, Anaise J et al. The effect of sodium monofluorophosphate and sodium fluoride on bone immobilization in rats. Calcif Tissue Res 1970; 5(2): $146-152$.

25 Messer HH, Armstrong WD, Singer L. Fluoride, parathyroid hormone and calcitonin: effects on metabolic processes involved in bone resorption. Calcif Tissue Res 1973; 13(3): 227-233.

26 Englander HR, Kesel RG, Gupta OP. The Aurora-Rockford, III., Study II. Effect of natural fluoride on the periodontal health of adults. Am J Public Health 1963; 53 : 1233-1242.

27 Taylor ML, Boyde A, Jones SJ. The effect of fluoride on the patterns of adherence of osteoclasts cultured on and resorbing dentine: a 3-D assessment of vinculinlabelled cells using confocal optical microscopy. Anat Embryol (Berl) 1989; 180(5): 427-435.

28 Den Besten PK, Yan Y, Featherstone JD et al. Effects of fluoride on rat dental enamel matrix proteinases. Arch Oral Biol 2002; 47(11): 763-770.

29 Hall TJ, Chambers TJ. Molecular aspects of osteoclast function. Inflamm Res 1996; 45(1): 1-9.

30 Drake FH, Dodds RA, James IE et al. Cathepsin K, but not cathepsins B, L, or S, is abundantly expressed in human osteoclasts. J Biol Chem 1996; 271(21): $12511-$ 12516.

31 Gowen M, Lazner F, Dodds R et al. Cathepsin K knockout mice develop osteoporosis due to a deficit in matrix degradation but not mineralization. J Bone Miner Res 1999; 14(10): 1654-1663.

32 Everts V, Korper W, Hoeben KA et al. Osteoclastic bone degradation and the role of different cysteine proteinases and matrix metalloproteinases: differences between calvaria and long bone. J Bone Miner Res 2006; 21(9): 1399-1408.

33 Oguro A, Kawase T, Orikasa M. NaF induces early differentiation of murine bone marrow cells along the granulocytic pathway but not the monocytic or preosteoclastic pathway in vitro. In Vitro Cell Dev Biol Anim 2003; 39(5/6): 243-248.

34 Oshita K, Yamaoka K, Udagawa N et al. Human mesenchymal stem cells inhibit osteoclastogenesis through osteoprotegerin production. Arthritis Rheum 2011; 63(6): 1658-1667.

(C) $\Theta$ This work is licensed under a Creative Commons Attribution-NonCommercialcc. ${ }_{\mathrm{BY}} \mathrm{NC}$ ND NoDerivs 4.0 Unported License. The images or other third party material in this article are included in the article's Creative Commons license, unless indicated otherwise in the credit line; if the material is not included under the Creative Commons license, users will need to obtain permission from the license holder to reproduce the material. To view a copy of this license, visit http://creativecommons.org/licenses/by-nc-nd/4.0/ 